\title{
GROUND-BASED \& SPACEBORNE SAR INTERFEROMETRIC TECHNIQUES SUPPORTING THE MANAGEMENT OF EMERGENCIES IN THE HEIMDALL PROJECT
}

\author{
J. A. Navarro *, G. Luzi, O. Monserrat, M. Crosetto \\ Centre Tecnològic de Telecomunicacions de Catalunya (CTTC/CERCA), Geomatics Division, Av. Gauss, 708860 Castelldefels, \\ Spain - (jose.navarro, guido.luzi, oriol.monserrat, michele.crosetto)@cttc.es
}

KEY WORDS: DInSAR, GB SAR, deformation, monitoring, multi-hazard, emergency, emergency management

\begin{abstract}
:
This paper describes the application of spaceborne and Ground Based radar interferometry as a tool for assisting the management of emergencies related to land movements. The support of the two techniques is carried out integrating some products, using tools available from a software and hardware architecture specifically developed within HEIMDALL, a H2020 project, devoted to providing a multi-hazard Cooperative Management, for Data Exchange, Response Planning and Scenario Building. Deformation maps obtained processing Sentinel-1 SAR images, updated every six days, can provide information over a large area, to be used during the preparedness and recovery phases. Data acquired through a Ground Based SAR system installed in-situ, provide a continuous and discontinuous (periodical) monitoring, aiming at supporting response and recovery phases. The products, in both cases, consist of deformation maps and temporal series, velocity of displacement, obtained through the application of Persistent Scatterer Interferometry (PSI).
\end{abstract}

\section{INTRODUCTION}

In this paper we report the application of radar interferometry, using both satellite and ground based SAR images, carried out within a project developed to provide a Multi-Hazard Cooperative Management Tool for Data Exchange, Response Planning and Scenario Building, namely the HEIMDALL H2020 European project.

The target of the ongoing H2020 HEIMDALL project (HEIMDALL Development Team, 2017) is to develop a system able to provide key stakeholders with the relevant tools to process data in the realm of emergency management, thus improving their response capacity. HEIMDALL's architecture is modular; its basic building block is a so-called Local Unit (LU). LUs are operated by individual authorities and offer response planning and scenario building capabilities (Figure 1).

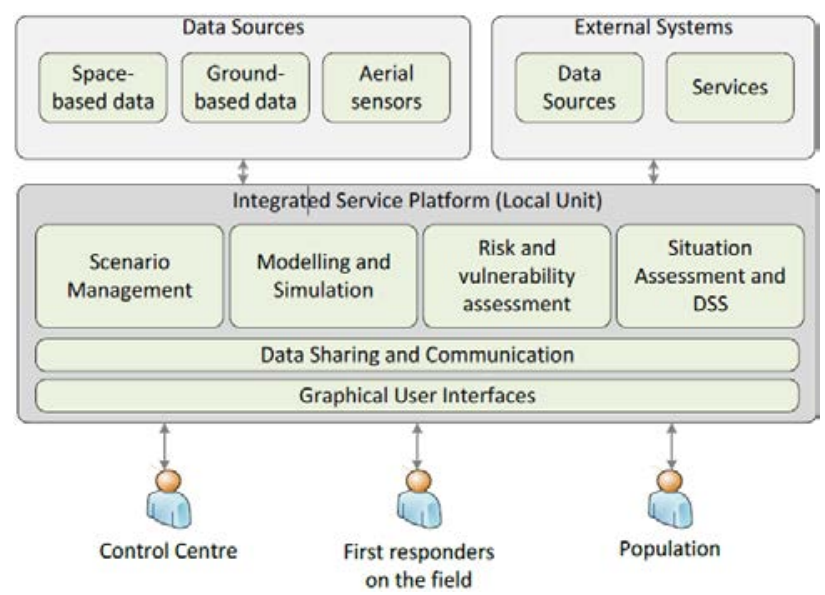

Figure 1: The HEIMDALL local unit (LU). Source: (HEIMDALL consortium, 2017)
HEIMDALL considers two different phases in the emergency management cycle, so the services it offers are separated accordingly. These are (a) the preparedness and mitigation and (2) response and recovery phases.

Users working in the first case (preparedness and mitigation) can manage (define, store, share) a catalogue of realistic scenarios relying on their real experience in the field. Such scenarios are shaped according to the specific requirements of several user profiles, such as firefighters, civil protection teams, police as well as command and control centres). The users may also link such scenarios to the response plans that, according to their knowledge and experience, should be applied to manage the emergency.

HEIMDALL, at this stage, considers only three kinds of hazards; these are forest fires, floods and terrain movements. The integration of satellite- and ground-based sensor data for these specific hazards in the system makes possible the provision of risk detection and monitoring services. Concerning the second situation (response and recovery), and once a risk or emergency has been detected, the system helps the decision-making process by means of hazard-specific modelling algorithms, decisionsupport tools and data-fusion techniques, so an improved assessment of the situation, its risk and vulnerability, is possible. Data coming from the real emergency is compared with the catalogue of scenarios, and then the most similar/ appropriate one may be thus selected, together with the related response plan and corresponding best practices.

Finally, all the information stored in the system - e. g., response plans, situation assessment data based on maps - may be accessed by all stakeholders as soon as it is available. To

\footnotetext{
* Corresponding author
} 
guarantee such instantaneous access to data - which is very important to first responders on the field - HEIMDALL makes use of a rapidly-deployable communications infrastructure to avoid the need to rely in existing communication networks, since these may have never existed, been destroyed or simply become unavailable because of the hazard. The use of such a portable, satellite-based communications system lets first responders on the field to access the information in the platform, provide data location, pictures - to store there as well as receiving any relevant messages generated by the platform (or its users).

HEIMDALL's architecture allows the federation of several LUs thus fostering data information sharing among stakeholders - that is, making possible the coordination of several emergency units in, for instance, cross-border disasters. This means that services, data and information may be accesses via a shared, global catalogue (Figure 2), thus increasing the flexibility of the system.

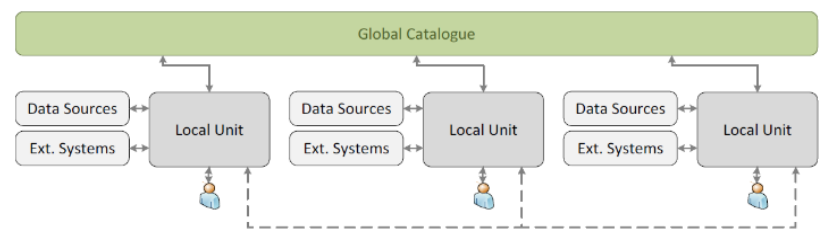

Figure 2: HEIMDALL Federation of Data and Services. Source: (HEIMDALL consortium, 2017).

Concerning data, HEIMDALL can deal with diverse external system and sources of these:

- External data and services. A multi-hazard management system as HEIMDALL cannot and may not ignore other already operational - even in the stage of development - systems. Providing a mechanism to incorporate these, makes HEIMDALL easily extendable; new services may thus be integrated, and new scenarios handled.

- Aerial-based data. The use of swarms of Micro Aerial Vehicles (MAV), "light drones", carrying a sensor platform also developed in the context of the project, is intended - but not limited to - monitoring and detection of fire hot spots. The Region of Interest (ROI) is partitioned and assigned to the members of the swarm, which inspect the assigned sub-areas autonomously.

- Space-based data. Space-based optical and radar sensors have been playing a vital role in large-scale disaster risk assessment and management - e.g., forecasting of thunderstorms and floods, monitoring of wildfires or damage assessment of landslides - by comparing imagery captured before and after the event. Data coming from these sensors are fed to into models able to analyse disaster-related developments - e.g., propagation of fire fronts or tsunami wave force intensity. The use of automatic processing chains, developed in recent years (Thomas et al. 2019) provide the operational basis to support authorities in dealing with disaster management and emergency response, especially in the case of wildfires, flooding or landslides. With the advent of the Sentinel fleet a new quality and extensiveness of data to support multi- hazard analysis process chains has become possible, making possible too the use of high-resolution data compiled in near real-time.

- Ground-based data. HEIMDALL uses in-situ sensors to monitor terrain movements such as landslides and subsidence. Activity and susceptibility maps rely on fixed information as geology, Digital Elevation Models (DEM), geomorphology and land cover. In addition, the deployment of sensors at the local level (hot spots) in areas with higher activity rates allows obtaining near real-time data (NRT), making possible the development of realistic scenarios and simulations for accurate response planning, as well as the management of the emergency during the crisis. The identification and tracking of key monitoring points are identified in the assessment phase and surveyed continuously. Ground Based Synthetic Aperture Radar (GB-SAR) and other techniques such as geodesic or topographic surveys are used to do this.

The next sections focus on the specific use of space- and groundbased data to help monitoring ground movements, and how the result of such process is integrated in the HEIMDALL platform

\section{PSI \& GB-SAR TECHNIQUES}

In general, the use of Space-based data for the monitoring of terrain movements risk in HEIMDALL is mainly based on the processing of two different data sets: optical and interferometric SAR imagery. In the first case, high resolution sensors with high spatial, spectral, and temporal resolutions can be used for all the three cases analysed in HEIMDALL: forest fires, floods and terrain movements. In this paper we focus only on SAR interferometry which is basically applied to the emergencies concerning terrain movements. The role of radar interferometry in the project is twofold. Firstly, it consists in applying the technique on Sentinel-1 satellite images to provide information over a large area, in the preparedness phase. The second case makes use of a GB SAR system installed in-situ, to provide a continuous and discontinuous (periodical) monitoring, aiming at supporting response and recovery phases respectively. The products in both cases consist of deformation maps and temporal series, velocity of displacement, obtained through the application of Persistent Scatterer Interferometry (PSI). Here below we resume in brief the main characteristics of these two approaches. Figure 3 shows a diagram of the location of the EO and GBSAR (included in the in-site sensors block) data, and how they flow into the HEIMDALL architecture.

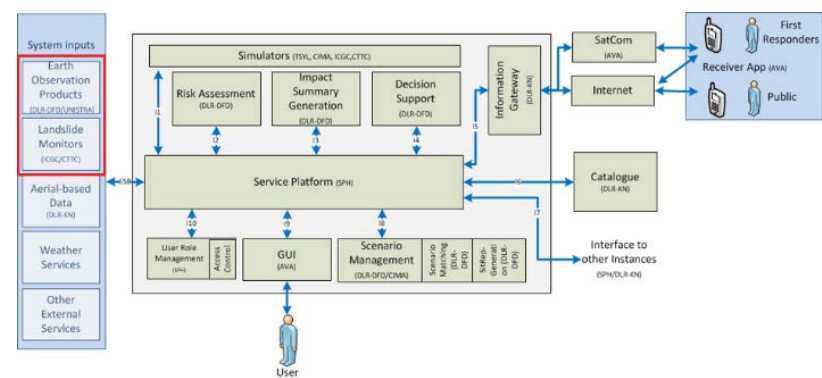

Figure 3: Scheme of the HEIMDALL project rationale. The red rectangle marks the Earth Observation and GB SAR data inputs to the Service Platform. Source: (HEIMDALL consortium, 2017). 


\subsection{PSI}

PSI represents a specific class of the Differential Interferometric Synthetic Aperture Radar (DInSAR) techniques, based on taking advantage of the information contained in the interferometric phase of at least two complex SAR images, acquired in different times over a common area. In the so-called repeat pass interferometry, the acquisition of the input images is carried out by the same sensor at different dates, dictated by the revisit time of the satellite; in the Sentinel-1 case this time shortest interval is 6 days. The measured phase is related not only to the deformation potentially occurred to the observed surface, but it contains some terms which must be estimated and separated from the deformation component. These are related to different factors: the effect of the different orbits and the local topography, mutable atmospheric conditions affecting the propagation of the radar signal in a different way during the different acquisitions, and the changes on the backscattering characteristics occurred in the time lapse between the different image acquisitions. These factors can introduce dramatic errors in the retrieval of the deformations. Persistent Scatterers (PS) are radar targets identified on the surface by the satellite which are identified by means of a numerical processing algorithm as good points for the retrieval of the deformation. The condition to assure that this process is effective, is that the point maintains a radar response, phase and amplitude, stable over time; this allows to perform displacement measurements with almost millimetre precision. The main outcomes of a PSI analysis include the deformation time series (TS) and the deformation velocity estimated over the analysed PSs. The higher is the density of these points, the better the reliability of the estimated deformation. Starting from clusters of PS, products for end users can be provided. In the case at hand a specific methodology has been applied, namely the Active Deformation Areas (ADA) algorithm developed at CTTC (Barra et al, 2017) as well as the tool that implements it (Navarro et al, 2018, Navarro et al, 2019), which will be detailed in the next section.

\subsection{Ground Based radar interferometry}

When the monitoring demands a continuous survey over smaller areas - e.g., corresponding to a single slope, or a village - to aid the response and recovery phase, GBSAR interferometry can be a valid tool also to integrate the presence of in situ sensors already present in the area. In this case the installation of a radar system, whose data can be updated every few minutes, can provide a continuous control. It is worth noting that the installation of a GBSAR system can be implemented in a few hours, allowing to make rapidly available to the stakeholders a safe, remote monitoring. To test the reliability of this technology within HEIMDALL rationale, a GBSAR system has been installed to acquire data from a scenario consisting of a small urban area including buildings, as well as debris due to a landslide; details about the main features of this radar system can be found in [Huang et al., 2017]. A picture of the setup is shown in Figure 4a.

The monitoring campaign was carried out focusing on a small village located in Northern Italy, at 1300 metres ASL namely Monesi di Mendatica, where a large landslide occurred in November 2016, destroying a main road and several buildings, and causing the evacuation of all the inhabitants. The GBSAR was installed in 2017, in a site in front of the area affected by the landslide, placed at approximately 300 metres from the scene, and at the same altitude; therefore, the elevation angle of the radar antennas is zero.
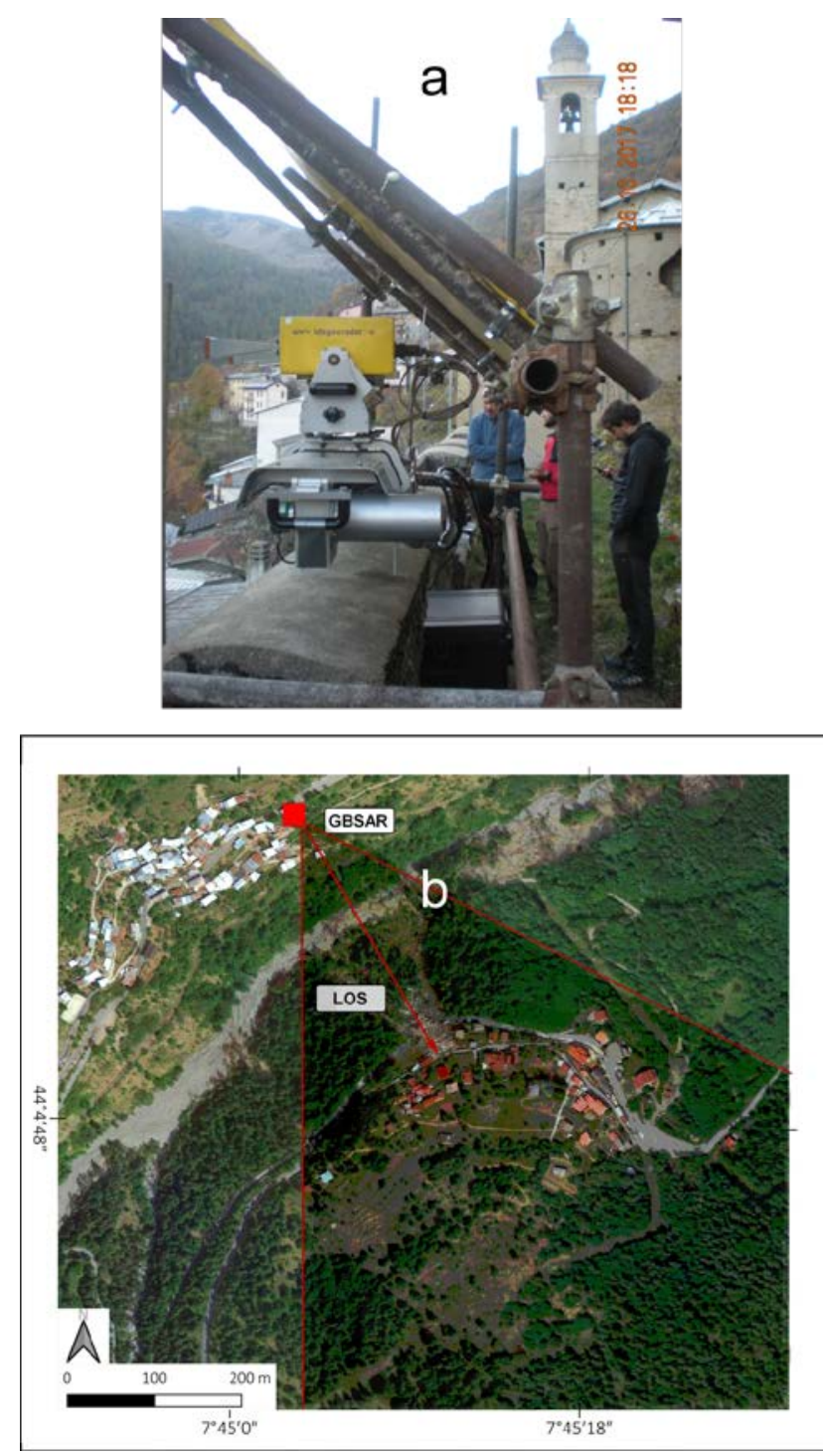

Figure 4: (a) picture of the GBSAR system in Monesi; (b) aerial view of the area of Monesi di Mendatica landslide with indicated the radar location, LOS direction and rough extension of the radar Field Of View (FOV).

Figure $4 \mathrm{~b}$ shows the orthophoto of the area monitored through the GBSAR. Figure 5a shows the amplitude radar image: some targets are marked to make easier to recognize the correspondence between the radar map and the real scene. Figure $5 \mathrm{~b}$ shows the area collapsed, composed of very heterogeneous material: e.g., bricks, rocks, roof pieces, undulated metal plates, soil. Examples of the products available form this monitoring tool are reported in the following section.

\section{SAR PRODUCTS FOR HEIMDALL}

\subsection{PSI}

The application of interferometric techniques to SAR data to detect and monitor the dynamics of landslides is well consolidated, and the achievement of the extent and the spatiotemporal evolution of instable slopes has been demonstrated in several papers and projects. 

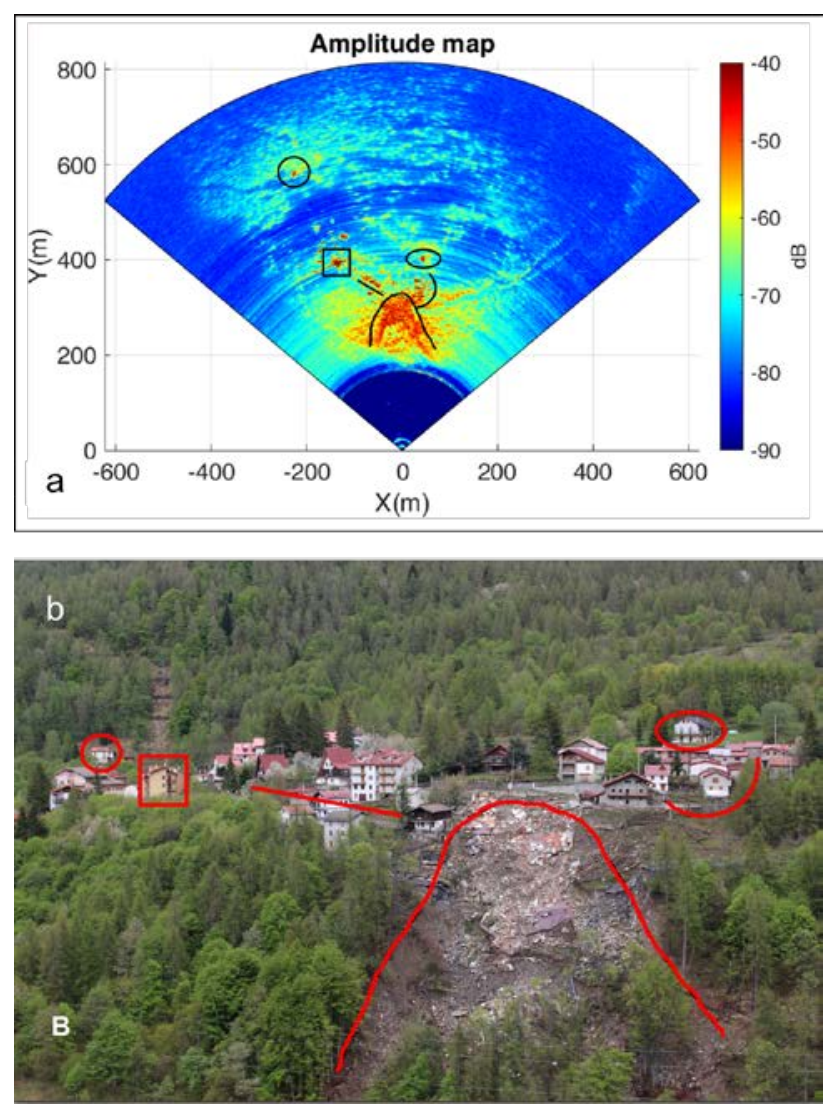

Figure 5: (a) Amplitude image of a GBSAR acquisition (VV pol) in radar coordinates, (b) photo of the main area of the landslide.

Examples of studies related to geohazard and subsidence can be found in (Crosetto et al., 2016). Several methodologies and processing tools have been developed. The characteristics of the Sentinel-1 constellation allow accomplishing long-term monitoring planning, at a regional scale, in any place of the world and free from data expenses. CTTC developed a specific processing methodology, to detect Deformation Activity Map (DAM) and the Active Deformation Areas (ADA) map (Barra et al, 2017, Navarro et al, 2018, Navarro et al, 2019).

The deformation activity map is the first result of the Sentinel-1 DInSAR processing; it consists in the estimated annual velocity and deformation time series (TS) in correspondence of selected points (PS). The DAM is the input to generate the Active Deformation Areas Map, which is derived by a semi-automatic extraction of the most significant detected active deformation areas (ADA). In Figure 6 the flowchart of the methodology developed to provide the products to HEIMDALL for the landslide detection and monitoring is depicted.

The developed methodology can be summarized in three main blocks:

- $\quad$ The Processing block which consists in the processing of Sentinel-1 data in order to obtain the preliminary deformation map.

- Post-processing block which consists in the simplification of the preliminary deformation map, in order to improve both, the reliability and the readability. The outputs of this block are the final
Deformation Activity Map and the Active Deformation Areas map.

- Geohazard management block which consists in the use of the ADA map together with other information in order to update the existing knowledge and derive useful maps for the geohazard management activities. The main output of this block is the Geohazard Activity Map (GAM) and the Vulnerable Element Activity Map (VEAM) (Barra et al. 2018).

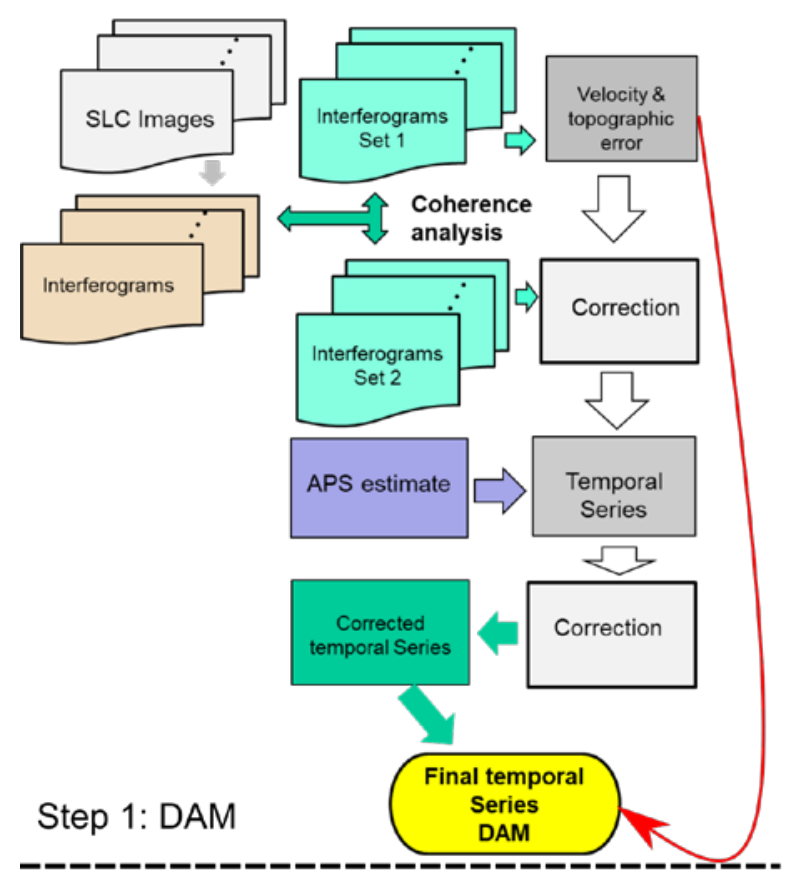

Step 2: ADA map

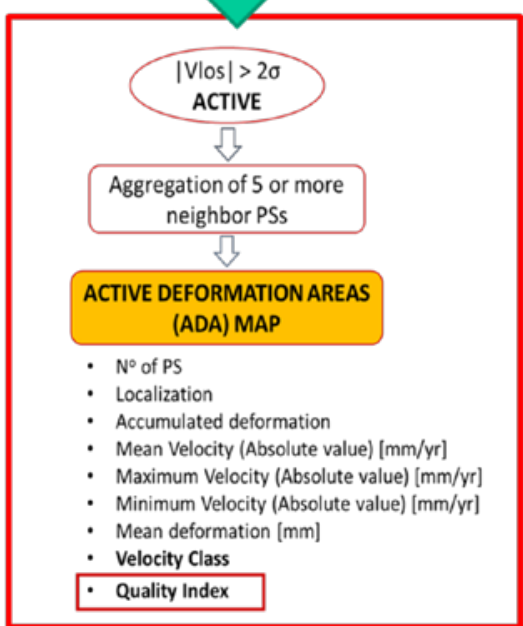

Figure 6: Workflow of the developed methodology. to produce DAM and ADA. The main products of the methodology are highlighted in red.

The basic spatial resolution of the Sentinel-1 products is $\sim 4$ by $14 \mathrm{~m}$ as provided by the Interferometric Wide swath (IW) mode. This tool makes also available, as a further product, a map where novel or active deformation areas are identified, as potential landslides. In order to periodically assess their activity state and 
update the existing Landslide Inventory Map, the output from the post processing activities are compared to the existing data inventory.

The produced maps and database are available in a specific web portal managed by CTTC, and accessible to the HEIMDALL concerned partners, through Web Map Service (WMS) or Web File Server (WFS) standards, to integrate the products in the HEIMDALL platform. Figure 7 depicts some examples of the kind of information shown by means of the HEIMDALL's Graphic User Interface (GUI). Both Line of Sight (LOS) velocity and ADA data correspond to the test area, Valle d'Aosta (Italy), selected for testing the methodology.
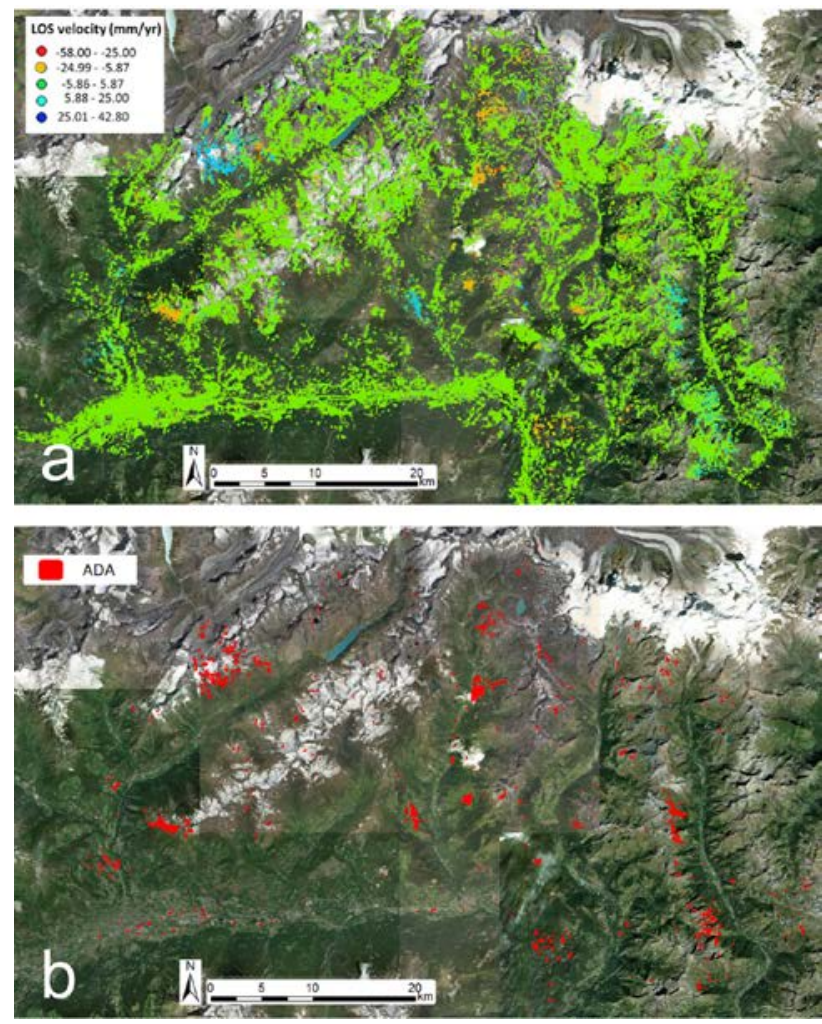

Figure 7: Example of ADA distribution in the Valle d'Aosta test site. (A) Line of Sight velocity (mm/year); (B) ADA map

\subsection{GBSAR}

Two main products are available from the GBSAR system: deformation maps and deformation temporal series. These products are linked to the HEIMDALL's Service Platform (SP) according to the scheme illustrated in Figure 8. This allows having complete information available both locally and at the decision centres.

This organization allows at the same time to analyse the data. In Figure 9 (on the next page) we show and example of the deformation map obtained at Monesi, through the HEIMDALL GUI.

\section{CONCLUSIONS}

This paper describes the application of a processing tool based on the interferometric radar processing of satellite and ground based interferometric radar images, aimed at supporting the early warning and the management of emergencies related to terrain movements.
1) Radar system

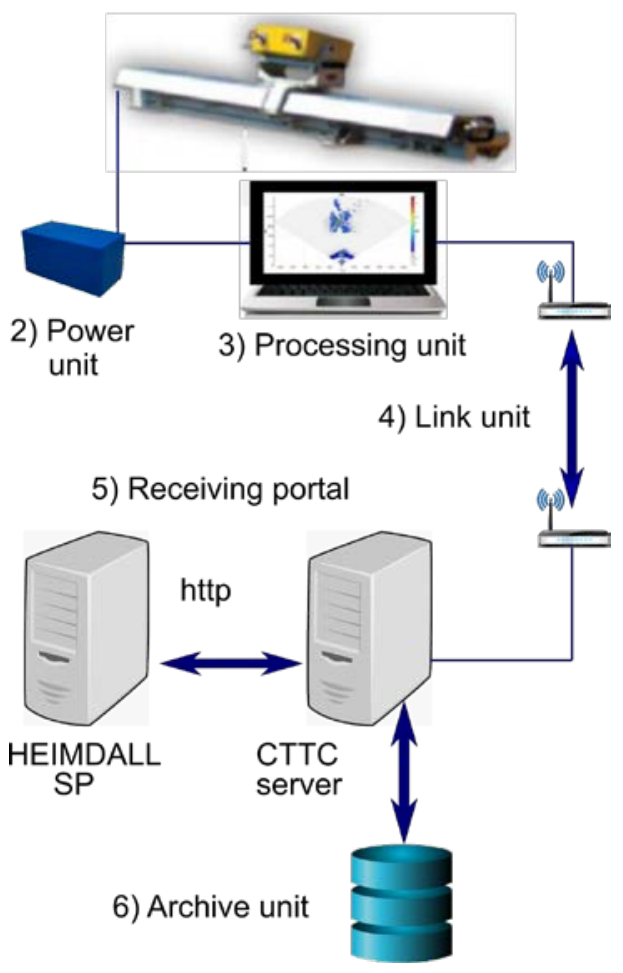

Figure 8: scheme of the connection and data link between the local radar station and the HEIMDALL SP.

The provided products, consisting in deformation maps and temporal series have been integrated into the architecture of the European H2020 project HEIMDAL. From one hand, and thanks to its high temporal resolution - just a few minutes - GBSAR, demonstrated to be a reliable tool to assist response and recovery phases providing both continuous and discontinuous monitoring data. On the other hand, the almost weekly revisit time of the satellite sensor acquisitions, fits better to risk assessment in the preparedness and mitigation phases.

\section{ACKNOWLEDGEMENTS}

This work has been partially financed by the European Union's Horizon 2020 research and innovation programme, with grant agreement No 740689, project HEIMDALL, "Multi-Hazard Cooperative Management Tool for Data Exchange, Response Planning and Scenario Building”. Thanks are due to Geohazard Monitoring Group, Institute of Research for Hydro-Geological Protection (IRPI), National Council of Research, Torino, Italy and to the Department of Earth Science and Environment, University of Pavia, Pavia, Italy, for their significant part in the Monesi GBSAR data acquisition and processing.

\section{REFERENCES}

Barra, A, Monserrat, O., Solari, L., Herrera, G., López, C., Onori, R., Reichenbach, P., González-Alonso, E., Mateos, R. M., Bianchini, S., Crosetto, M. 2018. The safety project: Sentinel-1 for the management of geological RISK, www.witpress.com, ISSN 1743-3533 (on-line) WIT Transactions on Engineering Sciences, Vol 121, (C)2018 WIT Press. doi:10.2495/RISK180221 2018. 
Barra, A., Solari, L., Béjar-Pizarro, M., Monserrat, O., Bianchini, S., Herrera, G., Crosetto, M., Sarro, R., González-Alonso, E., Mateos, R. M., Ligüerzana, S., López, C., Moretti, S., 2017. A Methodology to Detect and Update Active Deformation Areas Based on Sentinel-1 SAR Images. Remote Sens. 9, 1002.

Crosetto, M., Monserrat, O., Cuevas-González, M., Devanthéry, N., Crippa, B. Persistent Scatterer Interferometry: A review. ISPRS Journal of Photogrammetry and Remote Sensing 115, 7889.

HEIMDALL Consortium, 2017. Multi-Hazard Cooperative Management Tool for Data Exchange, Response Planning and Scenario Building. Horizon 2020 proposal number 740689, call H2020-SEC-2016-2017, topic SEC-01-DRS-2016.

HEIMDALL Development Team, 2017. Multi-Hazard Cooperative Management Tool for Data Exchange, Response Planning and Scenario Building. HEIMDALL consortium. heimdall-h2020.eu (3 May 2017).

Huang Q., Luzi, G., Monserrat, O, Crosetto, M. 2017. Groundbased synthetic aperture radar interferometry for deformation monitoring: a case study at Geheyan Dam, China, Journal of Applied Remote Sensing 11 (3), 0360302017.

Navarro, J.A., Cuevas, M., Barra, A., Crosetto, M., 2018. Detection of Active Deformation Areas based on Sentinel-1 imagery: An efficient, fast and flexible implementation. Proceedings of the 18th International Scientific and Technical Conference.

Navarro, J.A., Cuevas, M., Tomás, R., Barra, A., Crosetto, M., 2019. A toolset to detect and classify Active Deformation Areas using interferometric SAR data. Proceedings of the 5th International Conference on Geographical Information Systems Theory, Applications and Management, pp. 167-174.

Tomás, R., Pagán, J.I., Navarro, J.A., Cano, M., Pastor, J.L., Riquelme, A., Cuevas-González, M., Crosetto, M., Barra, A., Monserrat, O., Lopez-Sanchez, J.M., Ramón, A., Ivorra, S., Del Soldato, M., Solari, L., Bianchini, S., Raspini, F., Novali, F., Ferretti, A., Costantini, M., Trillo, F., Herrera, G., Casagli, N. Semi-Automatic Identification and Pre-Screening of GeologicalGeotechnical Deformational Processes Using Persistent Scatterer Interferometry Datasets. Remote Sens. 2019, 11, 1675.

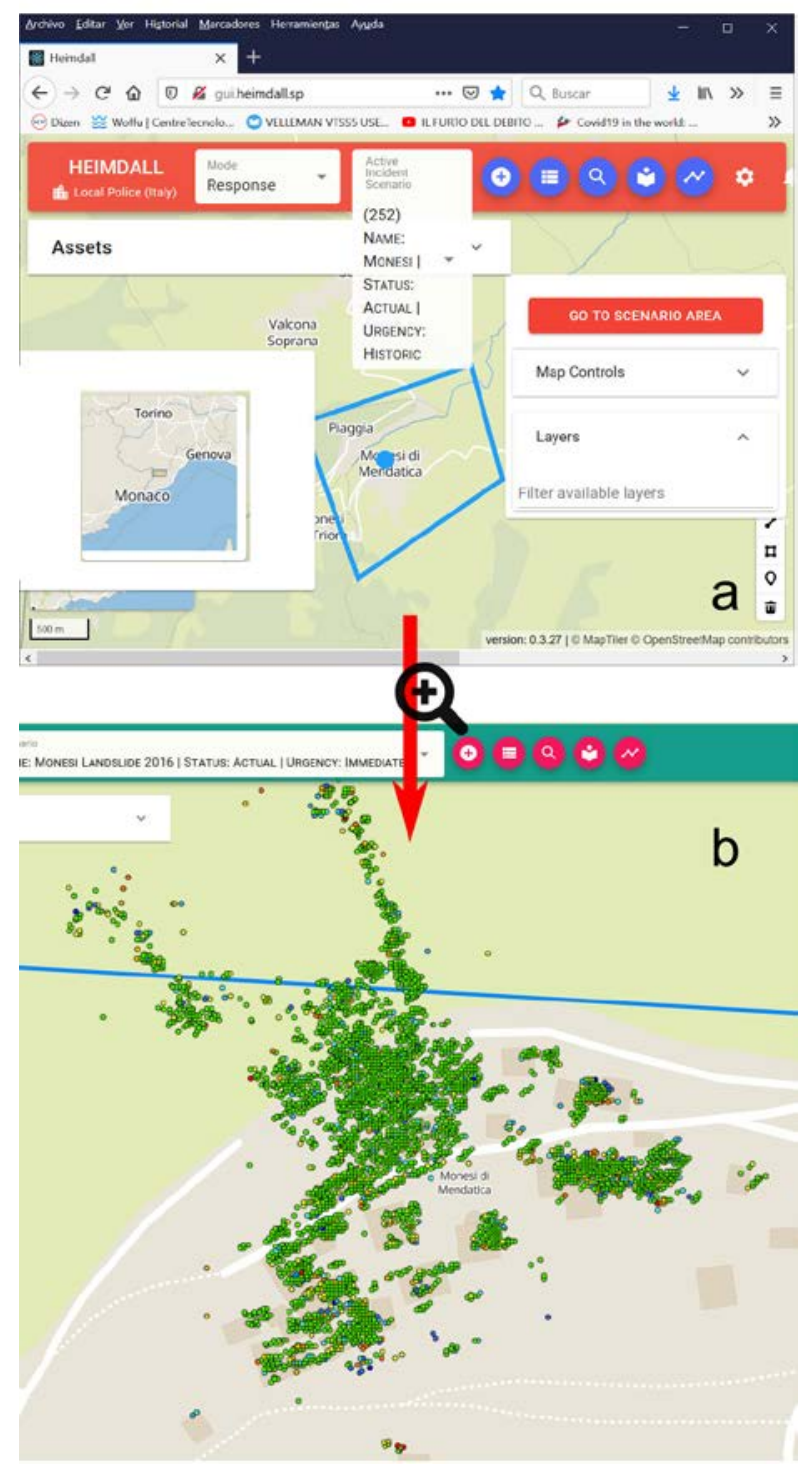

Figure 9: The web based HEIMDALL GUI (a) showing the Monesi test site area and (b) a zoomed scene depicting the deformation map for the same place. 